\title{
Shape-Preserving Half-Projective Warps for Image Stitching
}

\author{
Che-Han Chang ${ }^{1} \quad$ Yoichi Sato $^{2} \quad$ Yung-Yu Chuang ${ }^{1}$ \\ ${ }^{1}$ National Taiwan University ${ }^{2}$ The University of Tokyo
}

\begin{abstract}
This paper proposes a novel parametric warp which is a spatial combination of a projective transformation and a similarity transformation. Given the projective transformation relating two input images, based on an analysis of the projective transformation, our method smoothly extrapolates the projective transformation of the overlapping regions into the non-overlapping regions and the resultant warp gradually changes from projective to similarity across the image. The proposed warp has the strengths of both projective and similarity warps. It provides good alignment accuracy as projective warps while preserving the perspective of individual image as similarity warps. It can also be combined with more advanced local-warp-based alignment methods such as the as-projective-as-possible warp for better alignment accuracy. With the proposed warp, the field of view can be extended by stitching images with less projective distortion (stretched shapes and enlarged sizes).
\end{abstract}

\section{Introduction}

Image stitching is a process of combining a set of images into a larger image with a wider field of view of the scene. For robustness, image stitching is typically solved by finding global parametric warps to bring images into alignment [13]. Popular choices for the global warps include similarity, affine and projective ones. In spite of their robustness, global warps are usually not flexible enough for all types of scenes and motions. For example, the projective warp can only provide accurate alignment for planar scenes or parallax-free camera motions.

For addressing the model inadequacy of global warps and improving alignment accuracy, recently, several local warp models have been proposed, such as the smoothly varying affine (SVA) warp [9] and the as-projective-aspossible (APAP) warp [13]. For better alignment accuracy, rather than relying on a single global warp, these methods adopt multiple local parametric warps on the overlapping regions to account for misalignment. For the nonoverlapping regions, projective (affine) regularization is used for smoothly extrapolating warps beyond the image

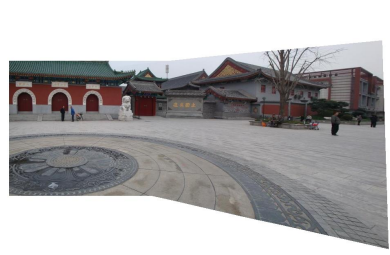

(a)

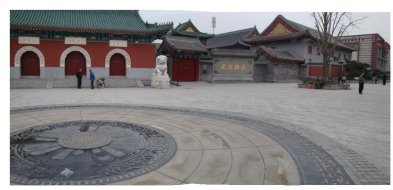

(c)

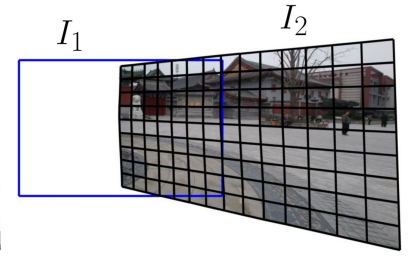

(b)

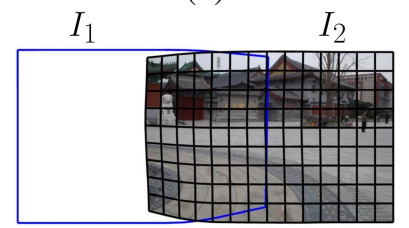

(d)
Figure 1. (a,b) APAP warp. (c,d) APAP + our warp. Note that the sizes of quads vary and their shapes distort in APAP (b), indicating severe size/shape distortion especially in the non-overlapping area of $I_{2}$. On the other hand, our warp maintains shapes and regularizes sizes of quads in (d), showing much less distortion.

overlap and resembling a global transform overall. For example, the APAP warp employs local projective warps within the overlapping regions while using moving DLT for smoothly extrapolating local projective warps into the non-overlapping regions. Thus, the result is a warp that is globally projective, yet allows local deviations to account for model inadequacy [13]. However, since the APAP warp attempts to resemble a projective warp globally, it suffers from the same problem as the projective warp: shape/area distortion, i.e., a part of the stitched image is severely stretched and non-uniformly enlarged.

Consider the traditional two-view stitching problem in Figure 1, in which two images $I_{1}$ and $I_{2}$ are stitched and $I_{1}$ is used as the base image. Figure 1(a) shows the result of the APAP warp [13]. The APAP warp locally adapts to different transformations, thus yielding more accurate alignment in the overlapping areas of two images. The warps in the overlapping areas are extrapolated into the non-overlapping areas and the resultant warp approximates a global projective warp (Figure 1(b)). As seen in this example, the non-overlapping area of $I_{2}$ is severely distorted in sizes and shapes by the projective warp. The distortion can be even 
aggravated when the next image is stitched with $I_{2}$ for further extending the field of view. Another thing worth note is, as pointed out by Hartley and Zisserman [7], applying the projective warp on $I_{2}$ effectively changes its perspective to $I_{1}$ 's. Thus, $I_{2}$ is warped as an extended view of $I_{1}$. The stitched image shows a wider perspective view of $I_{1}$ and thus is single-perspective. Cylindrical and spherical warps address the problem with a fairly narrow view of the perspective warp by providing multiperspective views. Unfortunately, these warps often curve straight lines and are only valid if all images are captured at the same camera center.

Since a single-perspective image with a wide field of view inevitably introduces severe shape/size distortion, the solution must provide a multiperspective stitched image. A preliminary idea is to employ a projective warp in the overlapping areas for better alignment while using a similarity warp in the non-overlapping areas for preserving the perspective of each view. We choose the projective warp because it is the most flexible global warp. On the other hand, we choose the similarity warp because it is composed of only translation, uniform scaling and rotation, and thus introduces no shape distortion nor non-uniform scaling. From another perspective, the similarity warp can be interpreted as a combination of panning, zooming and in-plane rotation of a camera, which keeps the viewing direction unchanged, thus preserving the perspective. Additionally, for constructing a smooth warp across the image, it is required to have a smooth transition between the region with the projective warp and the one using the similarity warp.

This paper proposes a novel parametric warp, the shapepreserving half-projective warp, which is a spatial combination of a projective transformation and a similarity transformation. Such a combination simultaneously takes into account the flexibility for alignment and the preservation of perspective. Given $I_{1}$ and $I_{2}$ in Figure 1, we first estimate the global projective transformation between them. From it, we determine a similarity transformation by a novel extrapolation scheme of the projective transformation. Intuitively, our warp performs a projective warp for alignment in the overlapping regions while preserving the image perspective of the non-overlapping areas with the similarity transformation. The extrapolation scheme is also used to form a smooth transition between areas with perspective and similarity warps. We also propose a scheme to integrate the proposed warp with the APAP warp. The combined warp aligns images in a local manner like the APAP warp, and generates a multiperspective view like the proposed warp. As shown in Figure 1(c)(d), the stitched image using the combined warp exhibits a multiperspective view while maintaining accurate alignment. With the proposed warp, the field of view can be extended without severe distortion on sizes, shapes and lines.

\section{Related Work}

Szeliski has a comprehensive survey of image stitching [10]. Conventional methods $[11,1]$ assume the camera motion contains only rotation. Image stitching is performed on a viewing sphere. A projection is performed to map the viewing sphere to an image plane for obtaining a $2 \mathrm{D}$ composite image. A few projection models have been proposed and they often aim to minimize the induced visual distortion due to projection. Brown et al. adopted the spherical projection in their AutoStitch program [1]. Zelnik-Manor et al. [14] used a multi-plane projection as an alternative to the cylindrical projection. Kopf et al. [8] proposed the locally adapted projection which is globally cylindrical while locally perspective. Carroll et al. [2] proposed the contentpreserving projection for reducing distortions of wide-angle images. When the underlying assumptions of these models are not met, misalignment occurs and post processing methods (e.g., deghosting and blending) can be used to hide it.

Image stitching techniques often utilize parametric transformations to align images either globally or locally. Gao et al. [6] proposed the dual-homography warping to specifically deal with scenes containing two dominant planes. The warping function is defined by a linear combination of two homographies with spatially varying weights. Since their warp is based on projective transformations, the resulting image suffers from projective distortion (which stretches and enlarges regions). They proposed an post-process to alleviate the distortion. Lin et al. [9] proposed a smoothly varying affine stitching field which is globally affine while allowing local deformations. Zaragoza et al. [13] proposed the as-projective-as-possible warp which is globally projective while allowing local deviations for better alignment.

Our approach is based on an analysis of projective transformations. Chum et al. [4] adopted a change of coordinates to derive a formula for computing geometric errors for projective transformations. Similarly, we use a change of coordinates to rephrase projective transformations. Chum and Matas [3] analyzed the local scale change under a projective transformation and utilized it for affine rectification. We follow the same analysis to design our warp.

\section{The proposed warp}

This section describes the proposed warping function $w: \mathbb{R}^{2} \rightarrow \mathbb{R}^{2}$ that maps $(x, y)$ to $\left(x^{\prime}, y^{\prime}\right)$. Given a projective transformation $\hat{H}$ relating two images spatially, we first divide $\mathbb{R}^{2}$ into two half-spaces $R_{H}$ and $R_{L}$ by a line. For $(x, y) \in R_{H}$, we let $w(x, y)=\hat{H}(x, y)$. For the other half space $R_{L}$, our warping function continuously extrapolates $\hat{H}$ to become a similarity transformation $S$. Since the proposed warp $w$ performs a projective transformation on one half-space while preserving shapes on the other half-space, we call it the shape-preserving half-projective warp. 


\subsection{Analysis of the projective transformation}

The construction of our warp comes from an analysis of the given projective transformation $\hat{H}$. A projective transformation (homography) $\hat{H}:(x, y) \mapsto\left(x^{\prime}, y^{\prime}\right)$ is defined as a linear transformation with homogeneous coordinates, i.e.,

$$
\left[\begin{array}{l}
x^{\prime} \\
y^{\prime} \\
1
\end{array}\right] \sim\left[\begin{array}{llc}
\hat{h}_{1} & \hat{h}_{2} & \hat{h}_{3} \\
\hat{h}_{4} & \hat{h}_{5} & \hat{h}_{6} \\
\hat{h}_{7} & \hat{h}_{8} & 1
\end{array}\right]\left[\begin{array}{l}
x \\
y \\
1
\end{array}\right]
$$

where $\sim$ denotes equality up to a scale factor. It has eight parameters. The mapping between $(x, y)$ and $\left(x^{\prime}, y^{\prime}\right)$ can be re-written as

$$
x^{\prime}=\frac{\hat{h}_{1} x+\hat{h}_{2} y+\hat{h}_{3}}{\hat{h}_{7} x+\hat{h}_{8} y+1}, \quad y^{\prime}=\frac{\hat{h}_{4} x+\hat{h}_{5} y+\hat{h}_{6}}{\hat{h}_{7} x+\hat{h}_{8} y+1} .
$$

Our analysis of $\hat{H}$ relies on a change of coordinates. The change simplifies the formula and reveals important properties. Chum et al. [4] adopted the same technique for computing the geometric error for a homography. In particular, we rotate the original coordinate system $(x, y)$ to form the new coordinate $(u, v)$. The new coordinate $(u, v)$ and the original coordinate $(x, y)$ are related as follows

$$
\begin{aligned}
{\left[\begin{array}{l}
x \\
y
\end{array}\right] } & =\left[\begin{array}{cc}
\cos \theta & -\sin \theta \\
\sin \theta & \cos \theta
\end{array}\right]\left[\begin{array}{l}
u \\
v
\end{array}\right], \\
\theta & =\operatorname{atan} 2\left(-\hat{h}_{8},-\hat{h}_{7}\right) .
\end{aligned}
$$

After the change of coordinates, we obtain a new homography $H$ that maps $(u, v)$ to $\left(x^{\prime}, y^{\prime}\right)$. The corresponding formula becomes

$$
\begin{array}{r}
{\left[\begin{array}{l}
x^{\prime} \\
y^{\prime} \\
1
\end{array}\right] \sim\left[\begin{array}{ccc}
h_{1} & h_{2} & h_{3} \\
h_{4} & h_{5} & h_{6} \\
-c & 0 & 1
\end{array}\right]\left[\begin{array}{l}
u \\
v \\
1
\end{array}\right],} \\
\text { where }\left[\begin{array}{ll}
h_{1} & h_{2} \\
h_{4} & h_{5}
\end{array}\right]=\left[\begin{array}{ll}
\hat{h}_{1} & \hat{h}_{2} \\
\hat{h}_{4} & \hat{h}_{5}
\end{array}\right]\left[\begin{array}{cc}
\cos \theta & -\sin \theta \\
\sin \theta & \cos \theta
\end{array}\right], \\
\left(h_{3}, h_{6}\right)=\left(\hat{h}_{3}, \hat{h}_{6}\right), \\
c=\sqrt{\hat{h}_{7}^{2}+\hat{h}_{8}^{2} .}
\end{array}
$$

The new mapping can also be written as

$$
\begin{aligned}
& x^{\prime}=H_{x}(u, v)=\frac{h_{1} u+h_{2} v+h_{3}}{1-c u}, \\
& y^{\prime}=H_{y}(u, v)=\frac{h_{4} u+h_{5} v+h_{6}}{1-c u} .
\end{aligned}
$$

We also denote the mapping as a function of $u$ and $v$, i.e., $\left[x^{\prime}, y^{\prime}\right]^{T}=H(u, v)=\left[H_{x}(u, v), H_{y}(u, v)\right]^{T 1}$.

\footnotetext{
${ }^{1}$ Without ambiguity, we use $H$ for both the transformation matrix and its corresponding mapping function.
}

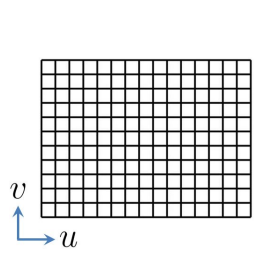

(a) Original

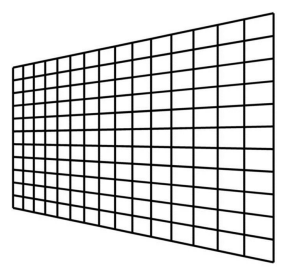

(b) Projective warp

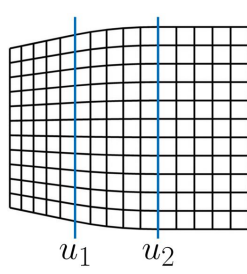

(c) Our warp
Figure 2. Comparison of the projection warp and our shapepreserving half-projective warp. Note that our warp exhibits much less shape and size distortion than the projective warp.

The main benefit of the change of coordinates is that the new projective transformation $H$ has its $h_{8}=0$ (Equation 5). Equivalently, note that now there is only one coordinate $u$ involved in the denominators of Equation 9 and 10 . The change of coordinates leads to the following properties:

(i) Change of scale. We first analyze how the scale changes across the image due to a projective transformation. The analysis relies on matrix decomposition. Specifically, $H$ can be decomposed into an affine transformation $H_{A}$ and a pure projective transformation $H_{P}$ as follows [3]

$$
\underbrace{\left[\begin{array}{ccc}
h_{1} & h_{2} & h_{3} \\
h_{4} & h_{5} & h_{6} \\
-c & 0 & 1
\end{array}\right]}_{H}=\underbrace{\left[\begin{array}{ccc}
h_{1}+c h_{3} & h_{2} & h_{3} \\
h_{4}+c h_{6} & h_{5} & h_{6} \\
0 & 0 & 1
\end{array}\right]}_{H_{A}} \underbrace{\left[\begin{array}{ccc}
1 & 0 & 0 \\
0 & 1 & 0 \\
-c & 0 & 1
\end{array}\right]}_{H_{P}} .
$$

The local area change of $(u, v)$ can be measured by the determinant of the Jacobian of $H$ at $(u, v)$, det $J(u, v)$, which is related with the determinants of the Jacobians of $H_{A}$ and $H_{P}$ as

$$
\begin{aligned}
\operatorname{det} J(u, v) & =\operatorname{det} J_{A}(u, v) \cdot \operatorname{det} J_{P}(u, v) \\
& =s_{A} \cdot \frac{1}{(1-c u)^{3}},
\end{aligned}
$$

where $s_{A}=\operatorname{det} J_{A}(u, v)$ is a constant independent of $u$ and $v$. From Equation 12, the scaling factor of the local area change induced by $H$ only depends on $u$. In particular, as the coordinate $u$ becomes larger, the local area through $H$ becomes larger, leading to larger area distortion. Figure 2(b) gives an example of a projective transformation, where the area distortion becomes larger along the positive $u$-axis.

(ii) Linearity of $H$. The affine transformation preserves the ratio of lengths on a line while the projective one does not in general. However, the change of coordinates reveals that, under certain conditions, the length ratios can be preserved under the projective transformation. From Equation 9 and 10, if $u=u_{0}$ is a fixed constant, then both $H_{x}\left(u_{0}, v\right)$ and $H_{y}\left(u_{0}, v\right)$ are linear functions of $v$,

$$
\begin{aligned}
& x^{\prime}=H_{x}\left(u_{0}, v\right)=\frac{h_{2}}{1-c u_{0}} v+\frac{h_{1} u_{0}+h_{3}}{1-c u_{0}}, \\
& y^{\prime}=H_{y}\left(u_{0}, v\right)=\frac{h_{5}}{1-c u_{0}} v+\frac{h_{4} u_{0}+h_{6}}{1-c u_{0}} .
\end{aligned}
$$


In other words, for a line parallel to the $v$-axis, $H$ preserves ratios of distances between points lying on that line.

(iii) Ruled surfaces. A ruled surface is a surface swept out by a moving line. It has a parameterization of the form $\mathbf{s}(u, v)=\mathbf{p}(u)+v \cdot \mathbf{r}(u)$ where $\mathbf{p}(u)$ is called the base curve and $\mathbf{r}(v)$ is called the director curve [5]. Consider a function $k$ of the form $k(u, v)=f(u) v+g(u)$, its graph (a surface formed by $(u, v, k(u, v))$ in $\left.\mathbb{R}^{3}\right)$ can be written as

$$
\left[\begin{array}{c}
u \\
v \\
k(u, v)
\end{array}\right]=\underbrace{\left[\begin{array}{c}
u \\
0 \\
g(u)
\end{array}\right]}_{\mathbf{p}(u)}+\underbrace{\left[\begin{array}{c}
0 \\
1 \\
f(u)
\end{array}\right]}_{\mathbf{r}(u)},
$$

showing that it is a ruled surface. Since $H_{x}(u, v)$ and $H_{y}(u, v)$ can be written in the form $f(u) v+g(u)$, their graphs are ruled surfaces. Equation 13 and 14 also reveal this fact. Equation 13 shows that the intersection of $H_{x}$ 's graph and the plane $u=u_{0}$ is a line, indicating that $H_{x}$ 's graph can be formed by moving and rotating a line along the $u$ axis. Thus, $H_{x}$ forms a ruled surface. The same argument applies to $H_{y}$ according to Equation 14 .

\subsection{Half-projective warp}

The construction of our warp makes use of the above three properties of the coordinate-changed projective transform $H$. First, we divide $\mathbb{R}^{2}$ by the line $u=u_{1}$ into two half spaces: $R_{H}=\left\{(u, v) \mid u \leq u_{1}\right\}$ and $R_{L}=\left\{(u, v) \mid u>u_{1}\right\}$. For $(u, v) \in R_{H}$, we apply the original transformation $H$. For $(u, v) \in R_{L}$, since $H$ causes larger area distortion on $R_{L}$ than $R_{H}$ (property(i)), we propose to replace $H$ with a similarity transformation $S$. The warp however must remain continuous; otherwise, there will be an obvious seam along the boundary of $R_{H}$ and $R_{L}$ in the warped image. To construct a continuous warp, we require that $S(u, v)=H(u, v)$ for all $(u, v)$ on the partition line. Note that such a requirement cannot be satisfied for an arbitrary partition line since the projective transform is not linear along a line in general while the similarity one is. Fortunately, by picking a line parallel to the $v$-axis, $u=u_{1}$, thanks to property(ii), the continuity requirement can be achieved because $H\left(u_{1}, v\right)$ is a linear function of $v$. By requiring $S\left(u_{1}, v\right)=H\left(u_{1}, v\right)$ for all $v, S$ is uniquely determined as

$$
S(u, v)=\frac{1}{1-c u_{1}}\left(\left[\begin{array}{cc}
h_{5} & h_{2} \\
-h_{2} & h_{5}
\end{array}\right]\left[\begin{array}{l}
u \\
v
\end{array}\right]+\left[\begin{array}{l}
\left(h_{1}-h_{5}\right) u_{1}+h_{3} \\
\left(h_{4}+h_{2}\right) u_{1}+h_{6}
\end{array}\right]\right) .
$$

Note that $u_{1}$ is a parameter of the warp. We discuss how to determine it in Section 4.

Figure 3 gives an example. Figure 3(a) shows the input image and the $u v$ coordinate system computed from the given projective warp $\hat{H}$. Figure 3(c) illustrates the constructed continuous half-projective warp using the above method. The construction gives us a warping function that is continuous $\left(C^{0}\right)$, half-projective and half-similarity.
Compared with a single projective transformation (Figure 3(b)), our warp has less shape and area distortion. However, it introduces a sudden line bending at $u=u_{1}$. More specifically, although lines lying within $R_{H}$ or $R_{L}$ are kept straight, lines passing through the partition line with $u=u_{1}$ are bent. To mitigate the artifacts, instead of using a single similarity transform in $R_{L}$, we introduce a buffer region where $H$ is smoothly extrapolated so that the warping function in $R_{L}$ is continuously differentiable $\left(C^{1}\right)$ and gradually changes from $H$ to a similarity transform $S$.

\section{3. $C^{1}$ extrapolation}

To construct a $C^{1}$ warp, we propose to generalize the above method by further dividing $R_{L}$ into two regions $R_{T}=$ $\left\{(u, v) \mid u_{1}<u<u_{2}\right\}$ and $R_{S}=\left\{(u, v) \mid u_{2} \leq u\right\}$ as shown in Figure 2(c). $u_{1}$ and $u_{2}$ are parameters of the warping function and will be determined in Section 4. The warping function is defined as

$$
w(u, v)=\left\{\begin{array}{ll}
H(u, v) & \text { if }(u, v) \in R_{H} \\
T(u, v) & \text { if }(u, v) \in R_{T} \\
S(u, v) & \text { if }(u, v) \in R_{S}
\end{array} .\right.
$$

$T(u, v)$ defined in the intermediate area $R_{T}$ is a function that gradually changes its behaviour from $H(u, v)$ to $S(u, v) . S(u, v)$ is a similarity transformation defined for $R_{S}$. We parameterize the similarity transformation by

$$
S(u, v)=\left[\begin{array}{l}
S_{x}(u, v) \\
S_{y}(u, v)
\end{array}\right]=\left[\begin{array}{cc}
\alpha & -\beta \\
\beta & \alpha
\end{array}\right]\left[\begin{array}{l}
u \\
v
\end{array}\right]+\left[\begin{array}{c}
t_{x} \\
t_{y}
\end{array}\right] .
$$

Given a projective transformation $H$, we first determine $u_{1}$ and $u_{2}$ and then construct a warp $w$ in the form of Equation 17.

From property(iii) in Section 3.2, we know that both graphs of $H_{x}$ and $H_{y}$ form ruled surfaces. From Equation 18, the graphs of $S_{x}$ and $S_{y}$ are planes in 3D which are also ruled surfaces. This motivates us to require that the graphs of $T_{x}(u, v)$ and $T_{y}(u, v)$ to be ruled surfaces as well. Therefore, we assume that $T(u, v)$ is of the following form

$$
T(u, v)=\left[\begin{array}{l}
T_{x}(u, v) \\
T_{y}(u, v)
\end{array}\right]=\left[\begin{array}{l}
f_{x}(u) \\
f_{y}(u)
\end{array}\right] v+\left[\begin{array}{l}
g_{x}(u) \\
g_{y}(u)
\end{array}\right] .
$$

For constructing the warp $w$, we need to determine the parameters, $\alpha, \beta, t_{x}, t_{y}$, for the similarity transformation $S$ and four functions $f_{x}, f_{y}, g_{x}, g_{y}$ for $T$. We assume these four functions are polynomial functions of $u$. Plugging Equation 9, 10, 18 and 19 into Equation 17, we can express the warping function $w$ as the following form:

$$
w(u, v)=\left[\begin{array}{l}
w_{x}(u, v) \\
w_{y}(u, v)
\end{array}\right]=\left[\begin{array}{l}
F_{x}(u) v+G_{x}(u) \\
F_{y}(u) v+G_{y}(u)
\end{array}\right],
$$




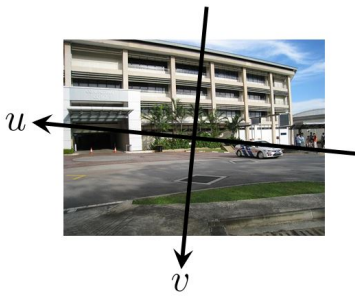

(a) Original

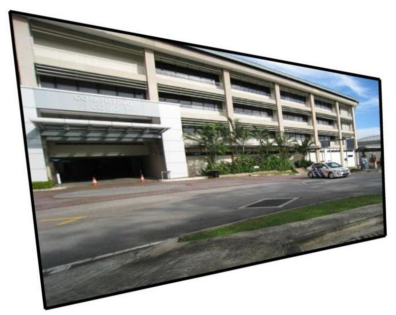

(b) Projective warp

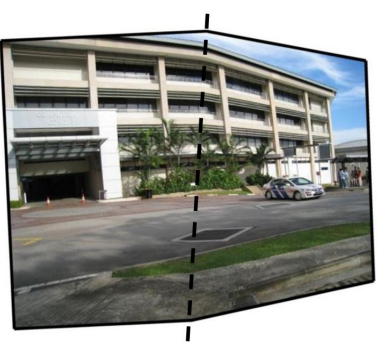

(c) Our $C^{0}$ warp

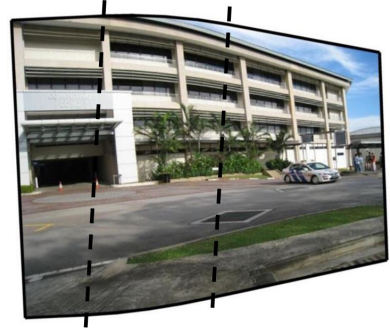

(d) Our $C^{1}$ warp

Figure 3. (a) The original image $I$ and the $u v$ coordinate system derived from $\hat{H}$. (b) The resultant image by warping $I$ with $\hat{H}$. (c) The resultant image by warping $I$ with our $C^{0}$ warp. (d) The resultant image by warping $I$ with our $C^{1}$ warp.

where

$$
\begin{aligned}
& F_{x}(u)= \begin{cases}\frac{h_{2}}{1-c u} & \text { if } u \leq u_{1} \\
f_{x}(u) & \text { if } u_{1}<u<u_{2} \\
-\beta & \text { if } u_{2} \leq u\end{cases} \\
& G_{x}(u)= \begin{cases}\frac{h_{1} u+h_{3}}{1-c u} & \text { if } u \leq u_{1} \\
g_{x}(u) & \text { if } u_{1}<u<u_{2} \\
\alpha u+t_{x} & \text { if } u_{2} \leq u\end{cases}
\end{aligned}
$$

$F_{y}$ and $G_{y}$ can be expressed similarly.

The remaining problem is to find proper $f_{x}, g_{x}, f_{y}, g_{y}$, $\alpha, \beta, t_{x}$ and $t_{y}$ so that $w$ is $C^{1}$ continuous. It requires that $F_{x}, G_{x}, F_{y}$ and $G_{y}$ are all $C^{1}$ continuous. Taking $F_{x}$ as an example, according to Equation $21, F_{x}$ is $C^{1}$ if the following four conditions are met,

$$
\begin{array}{ll}
f_{x}\left(u_{1}\right)=\frac{h_{2}}{1-c u_{1}} & \left(F_{x}\left(u_{1}\right) \text { is continuous }\right) \\
f_{x}^{\prime}\left(u_{1}\right)=\frac{c h_{2}}{\left(1-c u_{1}\right)^{2}} & \left(F_{x}^{\prime}\left(u_{1}\right) \text { is continuous }\right) \\
f_{x}\left(u_{2}\right)=-\beta & \left(F_{x}\left(u_{2}\right) \text { is continuous }\right) \\
f_{x}^{\prime}\left(u_{2}\right)=0 & \left(F_{x}^{\prime}\left(u_{2}\right) \text { is continuous }\right)
\end{array}
$$

Since we have four linear constraints, we can have at most four parameters. Because $\beta$ already takes one parameter, $f_{x}$ can have three parameters. Thus, we assume that $f_{x}$ is a quadratic function of $u$. By solving the resultant 4-by-4 linear system, we obtain $f_{x}(u)$ and $\beta$. Similarly, $f_{y}(u)$ and $\alpha$ can be solved by enforcing $C^{1}$ continuity of $F_{y}$. Following the same strategy, constraints on $G_{x}$ give us $g_{x}(u)$ and $t_{x}$. Finally, $G_{y}$ gives us $g_{y}(u)$ and $t_{y}$.

In sum, the proposed warp extrapolates $H(u, v)$ into $R_{T}$ and $R_{S}$ by automatically determining the functions $T(u, v)$ and $S(u, v)$. To conclude, given a projective transformation $\hat{H}$ relating two input images, we construct a warping function $w$ that is $C^{1}$ continuous, projective in one half-space and continuously transfers into a similarity transformation in the other half-space. Figure 3(d) demonstrates the constructed warp. The next section discusses how to apply the proposed warp to image stitching and determine parameters $u_{1}$ and $u_{2}$.

\section{Image stitching}

This section presents how to adopt the proposed warp for image stitching. We first describe how it can be adopted for pairwise stitching and how to automatically determine the parameters $u_{1}$ and $u_{2}$. Then we present a novel scheme to combine the proposed warp with the as-projective-aspossible (APAP) warp [13] to have the strengths of both.

\subsection{Shape-preserving image stitching}

Given two images $I_{1}$ and $I_{2}$, suppose they are related by a homography $H_{12}$ that maps a point $\left(x_{2}, y_{2}\right)$ in $I_{2}$ to $\left(x_{1}, y_{1}\right)$ in $I_{1}$. As shown in Figure 4 , if $I_{2}$ is warped by $w$, $I_{1}$ should be warped by $w \circ H_{12}^{-1}$ in order to maintain the geometric relationship described by $H_{12}$. When $w=H_{12}$, it is reduced to applying $H_{12}$ to $I_{2}$ while fixing $I_{1}$. When $w=I, I_{2}$ is fixed and $I_{1}$ is warped by $H_{12}^{-1}$. We derive the proposed warp from $H_{12}$ as described in Section 3 and use it as $w$ in Figure 4 for stitching two images.

Next we describe how to automatically determine the parameters $u_{1}$ and $u_{2}$. Since one goal of the proposed warp is to maintain the perspective of each image, we want that each image undergoes a similarity transformation as much as possible. That is, we want that the constructed warp $w$ approaches a similarity transformation. To achieve this, we associate each image $I_{i}$ with a cost $E_{i}$ which measures the deviation of its warp function $w_{i}$ from the nearest similarity transformation in the Frobenius norm, i.e.,

$$
\begin{aligned}
& E_{i}\left(u_{1}, u_{2}\right) \\
& =\min _{a_{i}, b_{i}} \iint_{(x, y) \in \Omega_{i}}\left\|J_{i}\left(x, y ; u_{1}, u_{2}\right)-\left[\begin{array}{cc}
a_{i} & -b_{i} \\
b_{i} & a_{i}
\end{array}\right]\right\|_{F}^{2} d x d y
\end{aligned}
$$

where $\Omega_{i}$ is the rectangular domain of $I_{i} ; J_{i}\left(x, y, u_{1}, u_{2}\right)$ is the Jacobian matrix of $w_{i}$ evaluated at $(x, y)$. Since $u_{1}$ and $u_{2}$ are parameters of $w_{i}$, the Jacobian depends on $\left(u_{1}, u_{2}\right)$. In the case of stitching two images, the total energy $E\left(u_{1}, u_{2}\right)=E_{1}\left(u_{1}, u_{2}\right)+E_{2}\left(u_{1}, u_{2}\right)$ where $w_{1}=w \circ H_{12}^{-1}$ and $w_{2}=w(w$ is the constructed warp 


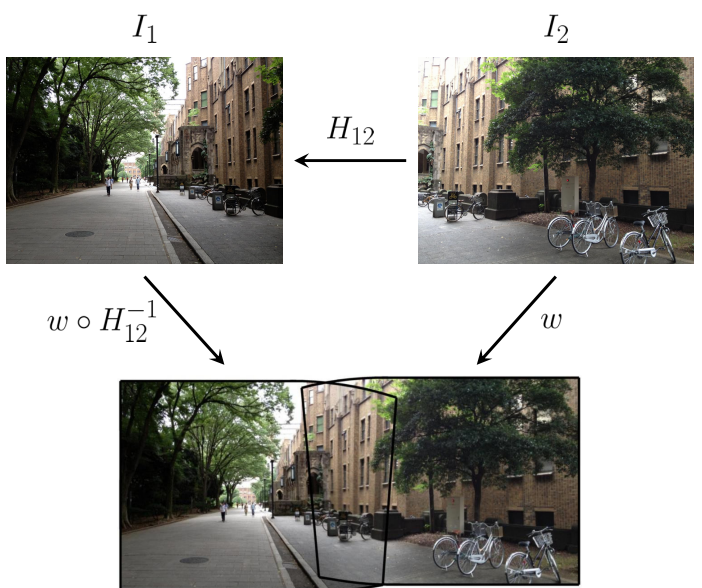

Figure 4. The geometric relationship between two images using the proposed warp.

from $H_{12}$ ). Note that, although minimizing individual $E_{i}$ could just lead to a similarity transform, their joint optimization finds a good compromise between the requirements of alignment and similarity. The energy is a nonlinear function of $u_{1}$ and $u_{2}$. We optimized it by regularly sampling the parameter space $\left(u_{1}, u_{2}\right)$, evaluating the energy at the sampled positions and picking up the one with the lowest energy.

For stitching an image sequence $\left\{I_{1}, \ldots, I_{n}\right\}$, we perform a group-wise stitching that simultaneously stitches all input images at once. Our method finds the homography $H_{i, i+1}$ for each pair of neighboring images and the homography $H_{1 n}$ between the first and the last image. $H_{1 n}$ is used for deriving a warp $w . I_{n}$ is warped by $w$ and each of other images $I_{i}$ is warped by $w \circ H_{i n}^{-1}$. Figure 5 shows an example that simultaneously stitches a sequence of three images.

\subsection{Combination with the APAP warp}

The proposed warp pays more attention on preserving perspectives but not alignment accuracy. However, it can be combined with approaches with better alignment accuracy to have strengths from both. The APAP warp [13] is one of the state-of-the-art image alignment methods in terms of alignment accuracy. One possible way for combining with APAP is to replace the projective warp in their moving DLT framework with the proposed half-projective warp. However, it would require determining spatiallyvarying $\left(u_{1}, u_{2}\right)$, which is nontrivial and unintuitive. In the following, we describe a simple scheme for combining the APAP warp with the proposed warp.

We interpret our warp as a two-stage process: a projective transformation $H$ followed by a post warp $w \circ H^{-1}$ as shown in Figure 6(a). This post warp can be treated as a refinement for reducing projective distortion. Specifically, our post warp only manipulates $R_{L}$ where there is large area

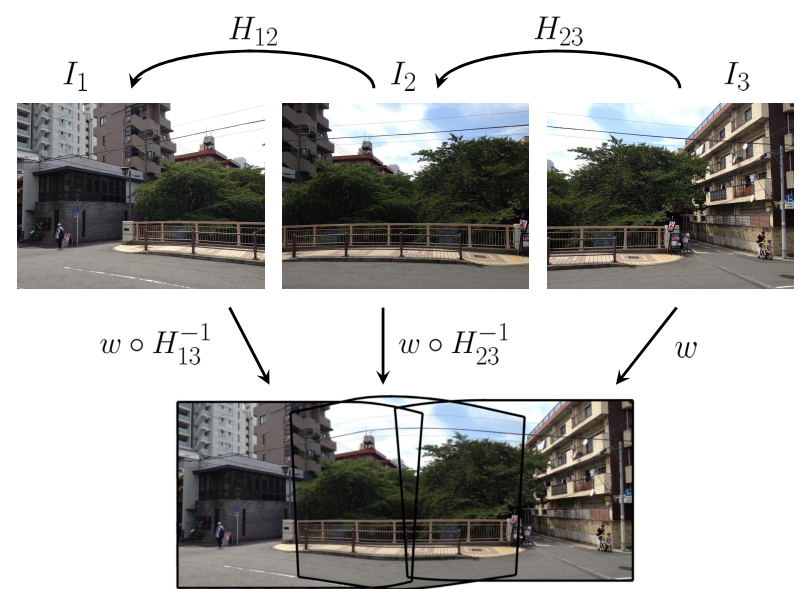

Figure 5. The geometric relationship among three images using our warp.

distortion while doing nothing on $R_{H}$. Although the APAP warp is a locally-varying warp, it follows a projective transformation globally. Thus, we can simply treat it as a projective warp and apply our post warp on it to reduce the distortion as shown in Figure 6(b). In this way, the images are locally aligned by APAP while the global shape is adjusted by our post warp. To summarize, the warping function that combines APAP and ours is defined as

$$
w \circ H^{-1} \circ w_{\text {APAP }} \text {. }
$$

Figure 6(c) shows the updated geometric relationship when adopting the combination of APAP and our warp.

\section{Experiments}

We compared our warp with the projective warp and AutoStitch [1]. AutoStitch assumes that input images share the same camera center and uses a 3D rotation model. For both the projective warp and our warp, we used the VLFeat library [12] to extract SIFT features and performed matching with RANSAC for obtaining the projective transformation for the overlapping regions. Stitching with the projective warp is achieved by fixing an image as the reference and applying the projective transformation on the other image. We manually chose the reference for the best result of the projective warp with less distortion. For our warp, after obtaining the projective transformation, we determined the parameters $u_{1}$ and $u_{2}$, constructed the warp and then applied the warping function to align images. The aligned images were composited using linear blending.

We implemented the proposed method using MATLAB and ran experiments on a $\mathrm{PC}$ with a $3.4 \mathrm{GHz} \mathrm{CPU}$ and $4 \mathrm{~GB}$ RAM. For stitching two images with the $800 \times 600$ resolution, our implementation took around 5 seconds for finding the parameters $u_{1}$ and $u_{2}$, and less than 2 seconds for both warp construction and image warping combined. 

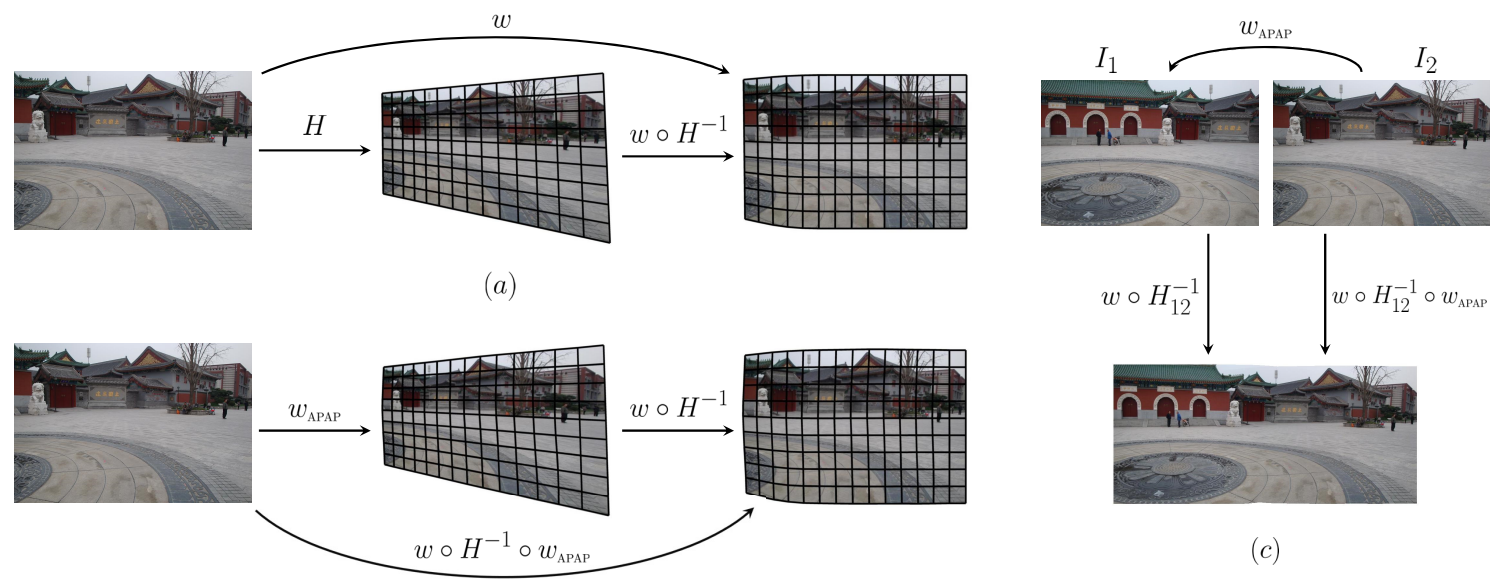

(c)

(b)

Figure 6. (a) Our warp can be interpreted as a projective warp followed by a post warp for refinement. (b) Integration by applying our post warp after the APAP warp. (c) The geometric relationship between two images using APAP+our warp.

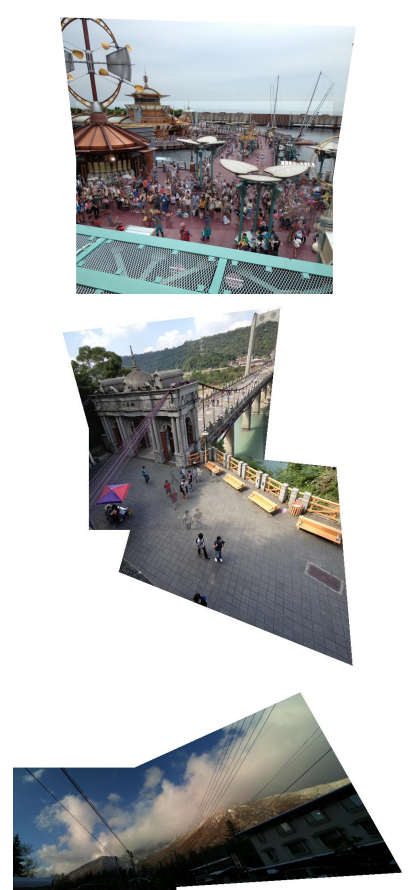

(a) Projective warp
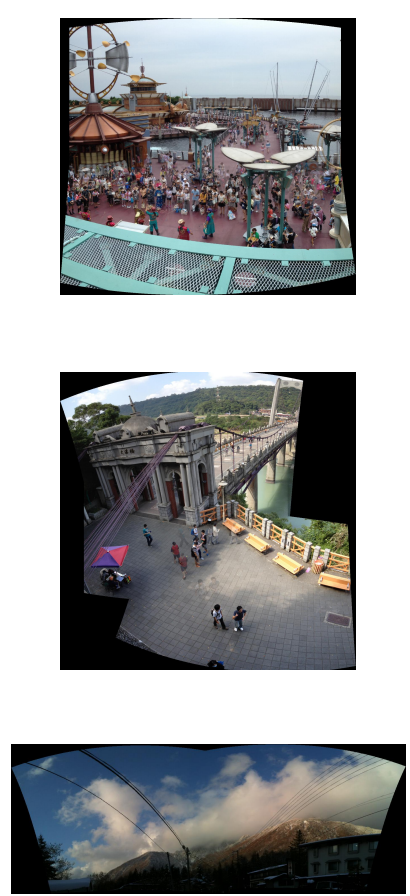

(b) AutoStitch
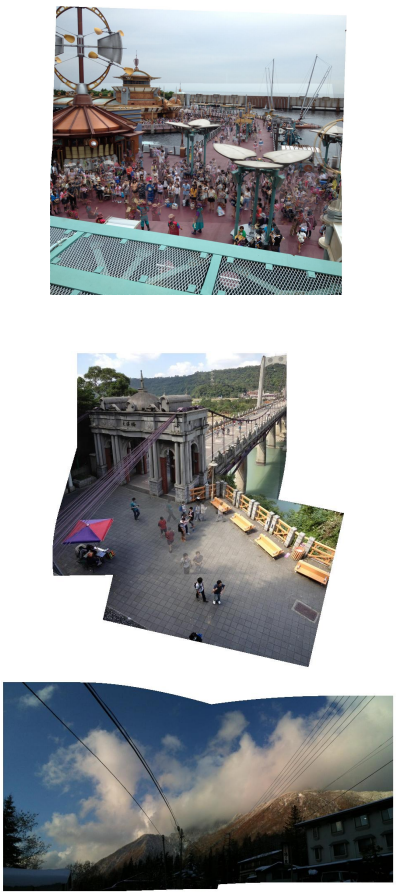

(c) Our warp

Figure 7. Comparisons of the projective warp, AutoStitch, and our warp.

Figure 7 compares the projective warp, AutoStitch and our warp. The projective warp keeps all lines straight, but exhibits projective distortion on sizes, shapes and orientations. AutoStitch can reduce area distortion. However, it curves some important lines. Our warp alleviates projective distortion while preserving the perspective of each image. Because both projective and similarity transformations do not introduce line distortion, our warp only suffers from line distortion within $R_{T}$. Figure 8 compares these three methods on stitching a sequence of ten images. AutoStitch introduces heavy shape distortion in this example. The pro- jective warp suffers from area and shape distortion particularly at both ends (the shapes of children are distorted). Our warp has much less distortion and provides a multiperspective view of the scene.

Figure 9 compares the APAP warp and the combination of APAP with our warp on two-image stitching. Our post warp is performed on both images by the warps described in Figure 6(c). Thus, our warp can maintain the accurate alignment as APAP does while adjusting the overall shape of the results for reducing distortion. Note that these examples can not be aligned well by single projective trans- 


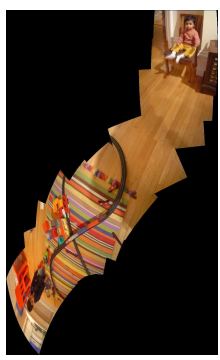

(a) AutoStitch

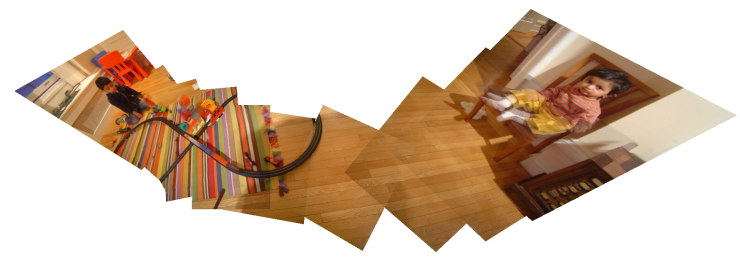

(b) Projective warp

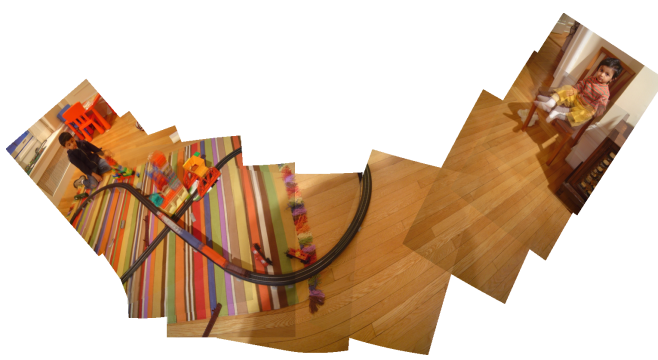

(c) Our warp

Figure 8. Comparisons of the AutoStitch, projective warp and our warp on an example of stitching 10 images.
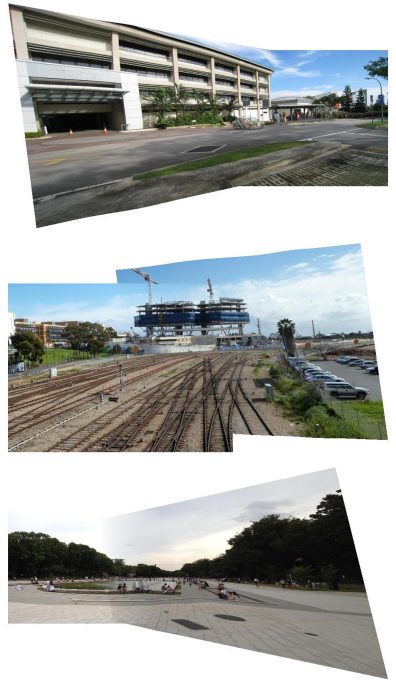

(a) APAP warp
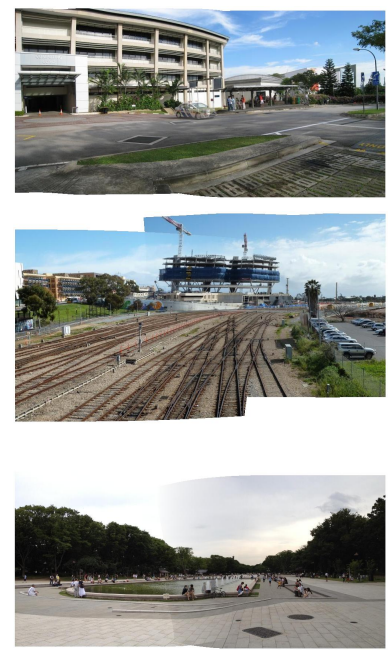

(b) APAP + our warp

Figure 9. Comparisons of the APAP warp and the combination of APAP and our warp.

formations. By combining with APAP, the proposed warp becomes more robust.

\section{Conclusion}

This paper proposes a novel warp for image stitching. Our warp aligns images globally while preserving their original perspectives. When combining with the APAP warp, the results provide accurate alignment, less distortion and multiperspective views. Our parameter selection procedure currently does not take image content (e.g., line features) into account. Therefore, when the scene is full of line structures, the selected parameters can be ineffective in reducing line distortion. In the future, we would also like to explore the possibility of adopting our warp to different applications such as view morphing.

Acknowledgement This work was partially done while Che-Han Chang was a visiting student, supported by the Interchange Association Japan (IAJ), at the University of Tokyo. This work was partly supported by grants NSC1012628-E-002-031-MY3 and NSC102-2622-E-002-013-CC2.

\section{References}

[1] M. Brown and D. G. Lowe. Automatic panoramic image stitching using invariant features. International Journal of Computer Vision, 74(1):59-73, 2007. 2, 6

[2] R. Carroll, M. Agrawal, and A. Agarwala. Optimizing content-preserving projections for wide-angle images. ACM Transactions on Graphics, 28(3):43, 2009. 2

[3] O. Chum and J. Matas. Planar affine rectification from change of scale. In Proceedings of ACCV 2010, pages 347360, 2011. 2, 3

[4] O. Chum, T. Pajdla, and P. Sturm. The geometric error for homographies. Computer Vision and Image Understanding, 97(1):86-102, 2005. 2, 3

[5] M. P. Do Carmo. Differential geometry of curves and surfaces. Prentice-Hall, 1976. 4

[6] J. Gao, S. J. Kim, and M. S. Brown. Constructing image panoramas using dual-homography warping. In Proceedings of IEEE CVPR 2011, pages 49-56, 2011. 2

[7] R. I. Hartley and A. Zisserman. Multiple View Geometry in Computer Vision. Cambridge University Press, second edition, 2004. 2

[8] J. Kopf, D. Lischinski, O. Deussen, D. Cohen-Or, and M. Cohen. Locally adapted projections to reduce panorama distortions. Computer Graphics Forum, 28(4):1083-1089, 2009. 2

[9] W.-Y. Lin, S. Liu, Y. Matsushita, T.-T. Ng, and L.-F. Cheong. Smoothly varying affine stitching. In Proceedings of IEEE CVPR 2011, pages 345-352, 2011. 1, 2

[10] R. Szeliski. Image alignment and stitching: A tutorial. Foundations and Trends in Computer Graphics and Vision, 2(1):1-104, 2006. 2

[11] R. Szeliski and H.-Y. Shum. Creating full view panoramic image mosaics and environment maps. In Proceedings of ACM SIGGRAPH 1997, pages 251-258, 1997. 2

[12] A. Vedaldi and B. Fulkerson. VLFeat: An open and portable library of computer vision algorithms. In Proceedings of ACM Multimedia 2010, pages 1469-1472, 2010. 6

[13] J. Zaragoza, T.-J. Chin, M. S. Brown, and D. Suter. Asprojective-as-possible image stitching with moving DLT. In Proceedings of IEEE CVPR 2013, pages 2339-2346, 2013. $1,2,5,6$

[14] L. Zelnik-Manor, G. Peters, and P. Perona. Squaring the circle in panoramas. In Proceedings of ICCV 2005, volume 2, pages 1292-1299, 2005. 2 